\title{
Haptic Hand For Heavy Duty Works In Industries
}

\author{
Akshay S. Tote ${ }^{1}$, Prof. R. K. Moje ${ }^{2}$, Rakesh U. Veri $^{3}$ \\ B.E. student, Department of Electronics Engineering, PDEA's College of Engg, Manjari (Bk.), Pune, India ${ }^{1,3}$ \\ Professor, Department of Electronics Engineering, PDEA's College of Engg, Manjari(Bk.), Pune, India ${ }^{2}$
}

\begin{abstract}
This paper presents an approach towards the Robotics field in a manner to give the solutions on problems related to limited working movements of the robots. The robots are used in a way to do the automation of the work which is done manually. The robots are generally used in various fields like Medical, Mechanical, Measurements and Instrumentation, Military and others. The Haptic technology can be implemented on the robots with minimal modifications on it. The robots can perform only work that they are programmed to do, but not other. With the help of Haptic technology, it can be possible to do the work which is different from the scheduled work of the robot.
\end{abstract}

Keywords: Automation, Degree of Freedom, flex sensor, Haptic, Accelerometer

\section{INTRODUCTION}

Robotic Arm is generally used in several applications such as manufacturing, surgery, handling hazardous objects, handling microscopic objects, and very heavy objects. The robotic hand which is working on Haptic technology is the most advanced version of the "Pick and Place" robot. In the industries, the people may have to work near hot zones or chemical zones or radioactive zones. For these purposes Haptic technology is extremely useful. A person from remote place moves his own hand to operate the robot to achieve the required work. Haptics is the scientific field that studies the sense of touch. We use motion sensors and can track the position and orientation of other objects with the help of this robotic hand, like our fingers. As a mouse or joystick can be used to control a computer, our finger actions could be used to control a program. These haptic controlled robots can be field(fixed) robots or movable robots.

The basic idea behind this project is a 'Sense' which is a thing that is used in the field of Sensors. The sensors senses the movements of our hands and the robot can work same as human hand to do the task which are not programmed in the robots.

The project is divided into two parts: Sensor side (Haptic Glove) and Receiver side (Robot).

\section{Sensor side:}

The glove fits over the user's hand which is fitted with the flex sensors on different joint over which we have to get the output. Also the accelerometer is used for movement of the whole robotic assembly in required direction. The joint may be fingers, wrist, elbow or shoulder.

\section{Receiver side:}

This part contains a fully working robot which outputs the result which is generated from robot controller as we perform the hand movements. As our hand has different joint, the robot also has the joints, but are known as "Degree of Freedom".

\section{LITERATURE SURVEY}

Haptic technology is used now-a-days in all possible fields in the world of technology. It may be medicine, automation, gaming, remote vehicle or in the interfaces made for blinds. Many medical surgeries are carried out with the help of haptics. Many game platforms are available with the haptic technology ready. Example can be given platforms use as Sony, Atari, Nintendo. These game many haptic devices to create a real time gaming environment. Also there are some science fiction movies which shows many imagined haptic techniques which may come to the real world in near future.

\section{RELATED WORK}

The haptic arm is made on this concept but it is working on AVR 8535 and using Zigbee for communication purpose which is costly. (International Journal of Latest Research in Science and Technology. ISSN (Online):2278-5299

Volume 2, Issue 2 : Page No.98-102, March - April (2013)).

The Virtual and Remote Laboratory which is used to educate the students about the robotics. With the help of this project the students can perform their practice on robotics form anywhere. (International Journal of Engg. Vol.22, no. 4, 2006).

The Haptic Rendering is used to "feel" the texture of an object in virtual environment. Also the work is going on the project which may be useful to "feel" the patterns of cloths virtually which are available on online shopping. (IEEE computer society. 0272-1716/04, March/April2004).

\section{PROPOSED SYSTEM DIAGRAM:}

\section{A. Sensor and Transmitter}

Working of Circuit:

\section{Haptic or Flex Sensors:}

Flex Sensor is used to sense bending moment. Flex sensors are the sensors that changes resistance depending 


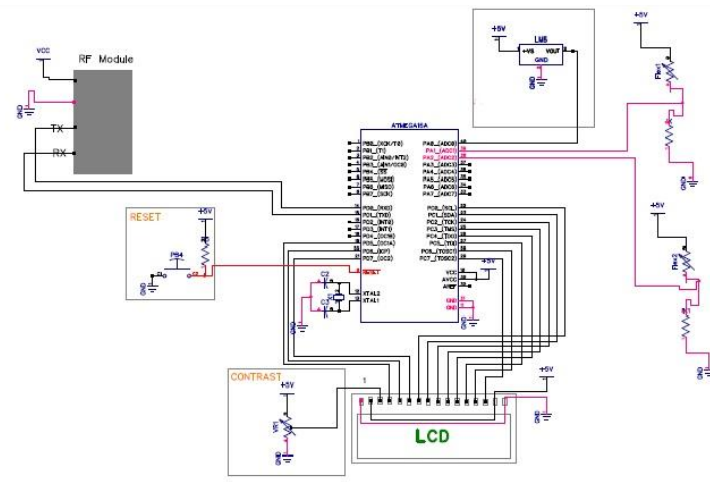

Figure 2: Sensor Side (Glove and sensors)

on the amount of bend on it. They convert change in bend into electrical resistance i.e. the more the bend, the more the resistance value. They can be made unidirectional or bidirectional. It is analog resistor. They work as analog voltage dividers. When the substrate is bent sensor produces a resistance output relative to the bend radius.

\section{Accelerometer}

We have used an accelerometer for the movement of whole robotic assembly in order to position the robot at any required place. ADXL335 from Analog Devices is a cost effective and easily available accelerometer. In place of push buttons on remote, we used accelerometer as we can control the robot on the hand positions only, no need to turn ON or OFF the buttons. So, we can control whole robot movements on single hand only. But for the ease we will control joint movements by one hand and the movement of whole robot assembly by another one.

\section{Microcontroller: ATmega16PU}

ATmega16 is an 8-bit high performance microcontroller of Atmel's Mega AVR family with low power enhanced RISC architecture with consumption. Atmega16 is based on (Reduced Instruction Set Computer) 131 powerful instructions. Most of the instructions execute in one machine cycle. Atmega16 can work on a maximum frequency of $16 \mathrm{MHz}$.

ATmega16 has $16 \mathrm{~KB}$ programmable flash memory, static RAM of $1 \mathrm{~KB}$ and EEPROM of 512 Bytes. ATmega 16 is a 40 pin microcontroller. There are $32 \mathrm{I} / \mathrm{O}$ lines which are divide into four 8-bit ports designated as PORTA, PORTB, PORTC \& PORTD.

\section{RF Transceiver:}

An RF module (radio frequency module) is usually a small electronic device used to transmit and/or receive radio signals between two devices. In an embedded system it is often desirable to communicate with another device wirelessly. This wireless communication may be accomplished through optical communication or through Radio Frequency (RF) communication. For many applications the medium of choice is RF since it does not require line of sight. RF communications incorporate a transmitter and/or receiver.

\section{Antenna:}

The receiver antenna picks up the serial data sent by the transmitter. Antenna is connected to one of the pins of the receiver. And this data is further processed through the receiver module.

\section{RF transceiver module-CC2500:}

CC2500 acts as a receiver module. It demodulates received data. This data is then sent serially to microcontroller (pin -14) on PD0 (RXD) pin.

\section{B. Receiver side (Robot):}

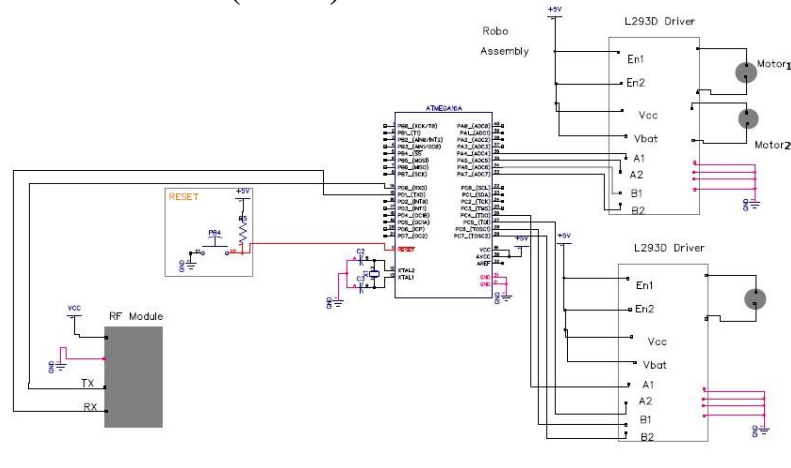

Figure 3: Receiver Side(Robotic Arm)

\section{Motor Driver}

L293D is a dual H-bridge motor driver integrated circuit (IC). Motor drivers act as current amplifiers since they take a low-current control signal and provide a higher-current signal. This higher current signal is used to drive the motors.

L293D contains two inbuilt H-bridge driver circuits. In its common mode of operation, two DC motors can be driven simultaneously, both in forward and reverse direction. The operations of two motors can be controlled by input logic at pins $2 \& 7$ and $10 \& 15$.

It works on the concept of $\mathrm{H}$-bridge. H-bridge is a circuit which allows the voltage to be flown in either direction. As you know voltage need to change its direction for being able to rotate the motor in clockwise or anticlockwise direction, hence H-bridge IC are ideal for driving a DC motor. In a single L293D chip there two $\mathrm{H}$-bridge circuit inside the IC which can rotate two dc motor independently. Due its size it is very much used in robotic application for controlling DC motors.

\section{DC Motor}

We are using high torque DC motors to operate the robotic arm. A DC motor is present at each joint. A DC motor relies on the fact that like magnetic poles repels and unlike magnetic poles attracts each other. A coil of wire with a current running through it generates an electromagnetic field aligned with the center of the coil. By switching the current on or off in a coil its magnetic field can be switched on or off or by switching the direction of the current in the coil the direction of the generated magnetic field can be switched $180^{\circ}$. 


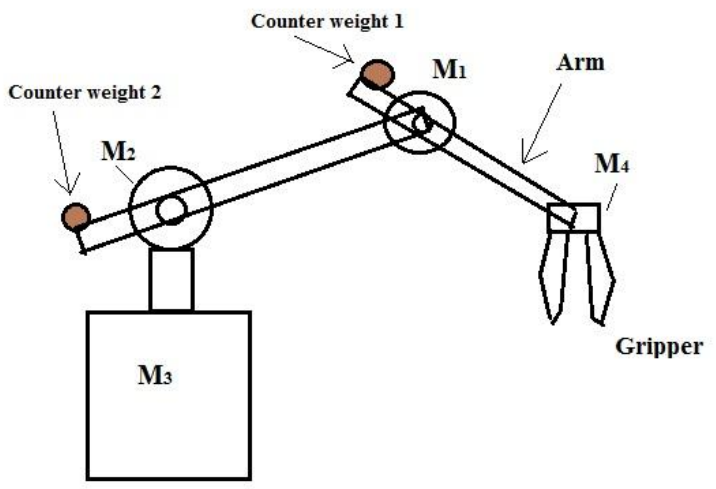

Figure no. 4: Schematic of DC Motor positions on Haptic Hand

\section{SOFTWARE USED:}

\section{AVR Studio}

We are using AVR Studio for programming the controllers. AVR Studio is a Development Tool for the AT90S Series of AVR microcontrollers. AVR Studio enables the user to fully control execution of programs on the AT90S In-Circuit Emulator or on the built-in AVR Instruction Set Simulator.

AVR Studio supports source level execution of Assembly programs assembled with the Atmel Corporation's AVR Assembler and C programs compiled with IAR Systems' ICCA90 C Compiler for the AVR microcontrollers. AVR Studio runs under Microsoft Windows95 and Microsoft Windows NT.

\section{DIPTRACE: PCB Design Software}

DipTrace PCB software is a software which can be used easily by students for their projects. As it is freely available with all the functionality of the full package, we have used this software for making the layout of PCBs. It includes an advanced automatic router that is able to route single- layer and multi-layer boards.

Auto router achieves high completion rates by going back and re-routing nets to make space for connections that could not be routed on a previous manual routing tools allow us to create traces by 90,45 deoree or without any limitations.

\section{PCB layout for Sensor side:}

Below shown figure is the PCB layout of sensor side for our project. This routing includes spacing for power supply, microcontroller, liquid crystal display, reset button. In high volume circuit board production, a bed of nails tester or fixture is used to make contact with the copper lands or holes on one or both sides of the board to facilitate testing.

Computers are used to control the electrical testing unit to send a mall current through each contact point on the bed of nails and verify that such current can be detected on the appropriate contact points.

\section{SCOPE OF THE PROJECT}

Future scope of the haptic technology covers a wide spectrum of human interaction with technology. Current research focuses on the mastery of the tactile interaction with holograms and distant objects, which if successful

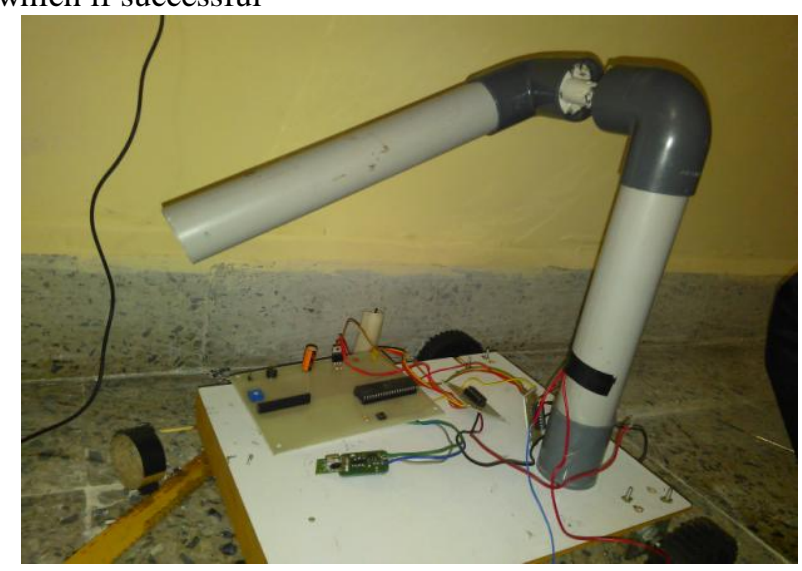

Fig. no. 5: Our project in progress

may result in applications and advancements in gaming, movies, manufacturing, medical, and other industries. The medical industry stands to gain from virtual and telescopic surgeries, which provides new options for medical care.

\section{RESULT}

The project runs successfully and the robotic arm is controlled easily through the hardware. We got the results as expected. This idea is ready to be installed on the industrial robots. So, if mistakenly any object falls from the gripper of any in-line robot, the work of that robot can be paused and through the sub-routine we can lift that object with haptics and the process can be resumed.

\section{CONCLUSION}

Our project may help the automotive, chemical \& radioactive industries to promote the automation at advanced level and will reduce human error and may help to human life to keep away from hazardous zones.

There may be a chance that clothing retail industry could gain from haptic technology by allowing users to "feel" the texture of cloths for sale on the internet. Future advancements in haptic technology may create new industries that were previously neither feasible nor realistic.

\section{REFERENCES}

11] Dr. Bhagwat S D., Gohil Vipul J., Nirmal Prateek R., Raut Amey P., "Robotics Arm Control Using Haptic Technology", International Journal of Latest Research in Science and Technology, Volume 2,Issue 2 : Page No.98-102, March - April

[2] Patnaik L. M., Varalakshmi B. D., Venugopal K. R., Thriveni J., "Haptics: State of the Art Survey", IJCSI International Journal of Computer Science Issues, Vol. 9, Issue 5, No 3, September 2012, ISSN (Online): 1694-0814

[3] Richard J. Adams, Blake Hannaford, "Stable Haptic Interaction with Virtual Environments", IEEE Transactions on Robotics and Automation, Vol. 15, No. 3, June 1999.

[4] Internet Sources: www.enginnersgarage.com www.ijcem.org www.worldhaptics.org 


\section{BIOGRAPHIES}

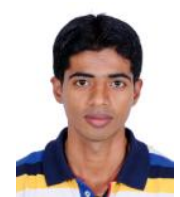

Rakesh U. Veri, student of B.E. Electronics having interest in Embedded Systems and Robotics. Have participated in different compititions of Robotics

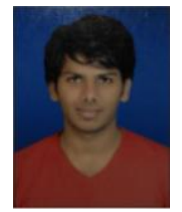

Akshay S. Tote, student of B.E. Electronics interested in Robotics studies and research in electronics field. 